\title{
Enhanced activity of massive black holes by stellar capture assisted by a self-gravitating accretion disc
}

\author{
V. $\operatorname{Karas}^{1}$ and L. Š $u b r^{2,3}$ \\ 1 Astronomical Institute, Academy of Sciences, Boční II 1401, 14131 Prague, Czech Republic \\ e-mail: vladimir.karas@cuni.cz \\ 2 Argelander Institut für Astronomie, University of Bonn, Auf dem Hügel 71, 53121 Bonn, Germany \\ 3 Charles University, Faculty of Mathematics and Physics, Astronomical Institute, V Holešovičkách 2, 18000 Prague, Czech Republic
}

Received 19 July 2006 / Accepted 15 April 2007

\begin{abstract}
Aims. We study the probability of close encounters between stars from a nuclear cluster and a massive black hole $\left(10^{4} M_{\odot} \lesssim M_{\bullet} \lesssim\right.$ $10^{8} M_{\odot}$ ). The gravitational field of the system is dominated by the black hole in its sphere of influence. It is further modified by the cluster mean field (a spherical term) and a gaseous disc/torus (an axially symmetric term) causing a secular evolution of stellar orbits via Kozai oscillations. Intermittent phases of high eccentricity increase the chance that stars become damaged inside the tidal radius of the central hole. Such events can produce debris and lead to recurring episodes of enhanced accretion activity.

Methods. We introduce an effective loss cone and associate it with tidal disruptions during the high-eccentricity phases of the Kozai cycle. By numerical integration of the trajectories forming the boundary of the loss cone, we determine its shape and volume. We also include the effect of a relativistic advance of the pericentre.

Results. The potential of the disc has the efffect of enlarging the loss cone, therefore, the predicted number of tidally disrupted stars should grow by factor of $\simeq 10^{2}$. On the other hand, the effect of the cluster mean potential, together with the relativistic pericentre advance, act against the eccentricity oscillations. In the end we expect the tidal disruption events to be approximately ten times more frequent in comparison with the model in which the three effects - the cluster mean field, the relativistic pericentre advance, and the Kozai mechanism - are all ignored. The competition of different influences suppresses the predicted star-disruption rate as the black hole mass increases. Hence, the process under consideration is more important for intermediate-mass black holes, $M_{\bullet} \simeq 10^{4} M_{\odot}$.
\end{abstract}

Key words. accretion, accretion disks - black hole physics - stellar dynamics

\section{Introduction}

According to the present-day consensus, the observational evidence shows black holes populating the mass spectrum in two distinct intervals: (i) stellar-mass black holes, $3 M_{\odot} \lesssim M_{\bullet} \lesssim$ $20 M_{\odot}$, that originate from collapse of massive stars and are revealed as members of binary systems; and (ii) supermassive black holes (SMBHs; $10^{6} M_{\odot} \lesssim M_{\bullet} \lesssim 10^{9} M_{\odot}$ ) hosted in the nuclei of many galaxies including our own. The existence of intermediate-mass black holes (IMBHs; $10^{2} M_{\odot} \lesssim M_{\bullet} \lesssim$ $10^{5} M_{\odot}$ ) is an open issue, as are their observational consequences and the form of the mass spectrum that might be filling the gap between the two well-established categories of black holes.

Over the past four decades, a picture of galactic nuclei has emerged in which black holes are embedded in a dense stellar system and their masses are increasing by accretion of gas from an accretion disc, gradually inflowing towards the horizon (Begelman \& Rees 1978; Volonteri \& Rees 2005). Remnants of stars tidally disrupted near an SMBH also contribute as a source of material (Zhao et al. 2002). Previously we demonstrated (Vokrouhlický \& Karas 1998; Šubr et al. 2004) that stars of the nuclear cluster can undergo episodes of very high orbital eccentricity if they interact with a self-gravitating disc near an SMBH. This mechanism sets stars on very elongated trajectories with small periapses, although the overall tendency of the gas-assisted drag acts in the opposite way - towards the orbital circularization (Syer et al. 1991; Vokrouhlický \& Karas 1998).
In this paper we study the fraction of stars that are set to highly eccentric orbits as a function of the black hole mass. We suggest that this problem is relevant in the context of the coexistence of a massive black hole with a surrounding cluster of stars. Exactly because of large elongation of the orbits, the fate of the remnant gas from the tidally disrupted stars is very uncertain, so we do not attempt to solve this problem in its entirety. Instead, we concentrate our attention on a single aspect - the mutual gravitational interaction of stars and the gaseous disc. To this aim, we study how Kozai's phenomenon modifies and enlarges the black-hole loss cone and how this change depends on $M_{\bullet}$. Our model is axially symmetric. We do take several subtleties of this scenario into consideration, namely the damping effect of the star cluster and the relativistic pericentre advance of stellar orbits near the central black hole. These effects pose a potential threat to our mechanism.

We find that a self-gravitating disc is capable of pushing more stars towards less massive (i.e. less-than-supermassive) black holes. Therefore, the effect should be particularly relevant for intermediate-mass black holes, provided they exist embedded within dense stellar systems and accrete from a gaseous disc or a torus (Madau \& Rees 2005; Miller \& Hamilton 2002).

There is a secondary motivation for our investigation: because the efficiency of the mechanism discussed here decreases with the increase in the black hole mass (it seems to be overwhelmed entirely by damping effects above $M_{\bullet} \sim 10^{7} M_{\odot}$ ), the present model may be particularly relevant to an ongoing debate 
about the origin of ultra-luminous X-ray sources (ULXs) and their putative connection with IMBHs (for reviews, see van der Marel 2003; Fabbiano 2006).

The ULXs are extra-nuclear, point-like X-ray sources with isotropic luminosities exceeding $10^{39} \mathrm{erg} \mathrm{s}^{-1}$. It has been proposed (Colbert \& Mushotzky 1999; Makishima et al. 2000; Matsumoto et al. 2001; Portegies Zwart et al. 2004; Hopman et al. 2004; Baumgardt et al. 2006) that some of them may harbour accreting IMBHs. Within the intermediate black-hole mass range, the proposed mechanism is relatively efficient, and we can thus speculate that these ULXs might be triggered when a star is damaged near the tidal radius. This concept seems to be in line also with the idea that accreting pre-galactic seed holes could indeed be detectable as ULXs (Madau \& Rees 2005). Also, it appears to be in accord with the manifestation of a multi-colour disc component, as reported in spectra of a sample of ULXs (Miller et al. 2004a,b; Fabian et al. 2004), and it also agrees with indications that ULXs are transient (Miniutti et al. 2006), possibly recurring phenomenon and that they are switched on during phases of active accretion (see Krolik 2004). However, the evidence for IMBHs is only circumstantial - numerous uncertainties persist in the mass estimates (see the discussion in King et al. 2001; Gonçalves \& Soria 2006), and so our scheme is a mere speculation at this stage.

\section{Model and method}

We assume that some kind of an accretion disc surrounds the central black hole and defines the equatorial plane of the system. The presence of the black hole provides a natural lengthscale that we use hereafter: $R_{\mathrm{g}} \equiv G M_{\bullet} c^{-2} \simeq 1.5 \times 10^{9} M_{4} \mathrm{~cm}$, $M_{4} \equiv M_{\bullet} /\left(10^{4} M_{\odot}\right)$. The disc is taken as axially symmetric and relatively light with respect to the central black hole $\left(\mu \equiv M_{\mathrm{d}} / M_{\bullet} \ll 1\right)$. Nevertheless, it is important that $\mu$ is greater than zero. To be specific, we consider two examples: (i) a constant surface density disc, characterised by its mass $M_{\mathrm{d}}$ and the outer radius $R_{\mathrm{d}}$; and (ii) a limiting case of an infinitesimally narrow ring of mass $M_{\mathrm{d}}$ and radius $R_{\mathrm{d}}$. The two cases serve as a useful test-bed because analytical expressions can be derived for the perturbation potential, $V_{\mathrm{d}}(R, z)$, and its first derivatives (Lass \& Blitzer 1983; Huré \& Pierens 2005). Notice also that the massive ring surrounding the central black hole can represent a time-averaged system with a secondary black hole, sometimes invoked in the framework of the hierarchical SMBH formation in centres of merging protogalaxies (Volonteri et al. 2003; Gültekin et al. 2004). Recently, Ivanov et al. (2005) applied the averaging technique to studying tidal disruptions of stars in a galaxy centre containing a supermassive binary black hole. However, these authors neglected relativistic effects, which exhibit growing importance near the black hole.

The central cluster is bound gravitationally to the black hole and characterised by distribution function on the space of osculating elements, $D_{\mathrm{f}}\left(a, C_{1}, e, \omega\right)$, where $C_{1}=\eta \cos i$ is a normalised $z$-component of the angular momentum, $\eta \equiv\left(1-e^{2}\right)^{1 / 2}$; $e$ is eccentricity, $a$ semimajor axis, and $\omega$ is the argument of pericentre. The orbit inclination $i$ is measured with respect to the disc plane. We consider distribution of the form

$D_{\mathrm{f}}\left(a, C_{1}, e, \omega\right)=K a^{1 / 4} e \eta^{-1}$

with $K$ being a normalisation constant. Equation (1) represents the Bahcall \& Wolf (1976) distribution with a random orientation in inclinations and linear distribution in eccentricities. The stellar cluster introduces a spherically symmetric perturbation to the central potential, $V_{\mathrm{c}}(r) \propto r^{1 / 4}$.
Lengthscales of the model can be related to the mass according to the empirical relation $M_{\bullet}(\sigma)$ (Tremaine et al. 2002), which we extrapolate in a naive way from galactic nuclei down to the IMBHs range: $M_{4} \simeq 10^{4} \sigma_{2}^{4}$, where $\sigma_{2} \equiv \sigma /\left(200 \mathrm{~km} \mathrm{~s}^{-1}\right)$ is the velocity dispersion. This seems to be justified by kinematical studies of some globular clusters (e.g., M 15, Gerssen et al. 2002; G1, Gebhardt et al. 2005; 47 Tuc, McLaughlin et al. 2006), and it enables us to introduce the cusp radius,

$R_{\mathrm{h}} \equiv G M_{\bullet} \sigma^{-2}=2.25 \times 10^{8} M_{4}^{-1 / 2} R_{\mathrm{g}}=0.11 M_{4}^{1 / 2} \mathrm{pc}$.

We further assume the mass of the cluster $M_{\mathrm{c}}\left(R_{\mathrm{h}}\right) \simeq M_{\text {. }}$. Finally, we set the disc/ring radius to be equal to the characteristic radius of the cluster: $R_{\mathrm{d}}=R_{\mathrm{h}}$. Thenceforth, only $M_{\mathrm{d}}$ is a free parameter.

The axial symmetry of the problem does not ensure conservation of the angular momentum; instead, only one of its components is conserved, so that the orbital eccentricity and inclination of the cluster stars can secularly evolve and fluctuate. In our model we imagine that Kozai's phenomenon ${ }^{1}$ is responsible for oscillations of the orbital elements on the time-scale of

$T_{\mathrm{K}} \equiv \frac{\lambda}{2 \pi \mu}\left(\frac{R_{\mathrm{d}}}{a}\right)^{3} P \simeq 1.6 \times 10^{3} M_{4} \frac{\lambda}{\mu}\left(\frac{R_{\mathrm{d}}}{a}\right)^{3}\left(\frac{a}{10^{8} R_{\mathrm{g}}}\right)^{3 / 2} \mathrm{yr}$.

The oscillation period exceeds the orbital period $P(a)$ of each individual star, $T_{\mathrm{K}} \gg P=2 \pi a^{3 / 2} /\left(G M_{\bullet}\right)^{1 / 2}$, by at least one order of magnitude. Factor $\lambda \simeq 1$ includes additional corrections arising from unaccounted effects.

Precession due to an extended spherically-symmetric potential component accelerates the oscillations. For $M_{\mathrm{c}} \gtrsim 0.1 M_{\text {e we }}$ find the correction term to the cycles of eccentricity oscillations:

$\lambda \simeq 0.1\left(\frac{M_{\mathrm{c}}}{M_{\bullet}}\right)^{-1 / 2}$.

The period $T_{\mathrm{K}}$ from Eqs. (3)-(4) fits well with results of the numerical integration shown in Fig. 1, where we evaluate the Kozai cycle period as a function of the mass $M_{\mathrm{c}}$ of the spherical cluster. The value of $T_{\mathrm{K}}$ in terms of orbital period $P$ is determined numerically by integrating the trajectories that reach the tidal radius $R_{\mathrm{t}}$ at the maximum of the eccentricity oscillations; i.e. $T_{\mathrm{K}}$ in Fig. 1 is given by the loss-cone boundary trajectories. The dependency indicates that the shape of the loss cone is also modified, so we look into this more in the next section.

\subsection{Loss cone in the presence of Kozai's process}

The elongated trajectories bring stars of the nuclear cluster close to the centre. It is then natural to assume that passages near the black hole are important moments in the evolution of the system by feeding the black hole and triggering the accretion activity, as is commonly described in terms of loss-cone processes (e.g. Frank \& Rees 1976; Magorrian \& Tremaine 1999; Merritt \& Wang 2005).

A "classical" loss cone is determined by the process of tidal disruption of stars near the central black hole. Low angular momentum trajectories are relevant because stars following these orbits have their periapses within the black hole tidal radius,

$R_{\mathrm{t}}=\left(\frac{M_{\bullet}}{M_{\star}}\right)^{1 / 3} R_{\star} \simeq 10^{3} M_{4}^{-2 / 3}\left(\frac{M_{\star}}{M_{\odot}}\right)^{-1 / 3}\left(\frac{R_{\star}}{R_{\odot}}\right) R_{\mathrm{g}}$,

1 Originally (Kozai 1962; Lidov 1962), the averaging method was applied to studying the long-term evolution in the context of the restricted three-body problem. Naturally, the approach can be readily applied to discussing the motion in an axially symmetric potential. 


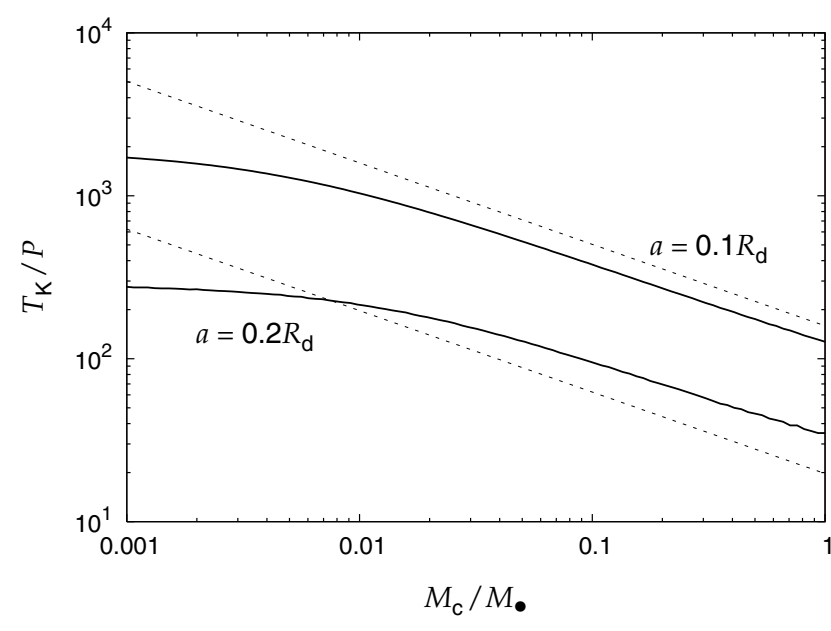

Fig. 1. Kozai period $T_{\mathrm{K}}$ (solid lines) as a function of mass distributed in the spherical potential of the nuclear cluster. Time is scaled with the corresponding orbital period $P$. Principal parameters of the system are the black hole mass, $M_{4}=10$, and the mass of the ring, $M_{\mathrm{d}}=0.1 M_{\bullet}$. Trajectories were characterised by $C_{1}=10^{-3}$ and integrated numerically. Two different values of semi-major axis are given by each curve. Also plotted is the approximation to $T_{\mathrm{K}}$ with the value of $\lambda$ from Eq. (4), producing the slope $-1 / 2$ (dotted lines).

where $M_{\star}$ and $R_{\star}$ are the stellar mass and radius. The loss cone is emptied on a time scale of $\simeq P$, provided the perturbing processes are not strong enough to deflect stars from their orbits.

This situation becomes more complicated when the time scales are modified in the presence of Kozai's mechanism. An "effective" loss cone can now be associated with the new period $T_{\mathrm{K}}>P(a)$ operating in the system. The loss cone is formed by trajectories in phase space plunging below $r=R_{\mathrm{t}}$ by Kozai's oscillations. This view of the influence of the process takes into consideration that orbital elements evolve in a systematic manner under Kozai's mechanism. In other words, eccentricities are pumped up to high values for extended periods of time, which makes the effect distinct from the stochastic nature of gravitational scattering. Furthermore, the loss-cone geometry is more complex because of the coupling that exists between the mean eccentricity and inclination of the orbits.

We characterise the influence of highly eccentric orbits on the star-capture rate by the fraction of stars that exhibit pericentre distances below a certain threshold radius, $R_{\mathrm{p}} \leq R_{\text {min }}$. We denote this fraction $\mathcal{F}_{2}\left(R_{\min } ; a, C_{1}\right)$ and write it in the form

$\mathcal{F}_{2} \equiv \frac{1}{D_{2}} \int_{0}^{\sqrt{1-C_{1}^{2}}} \mathrm{~d} e \int_{0}^{2 \pi} \mathrm{d} \omega \Theta\left(e_{\max }-e_{\min }\right) D_{\mathrm{f}}\left(a, C_{1}, e, \omega\right)$,

where $\Theta$ is the Heaviside step function, $e_{\min } \equiv 1-R_{\min } / a$, and $e_{\max }\left(a, C_{1}, e, \omega\right)$ is the maximum eccentricity reached on a given trajectory. The normalisation factor is equal to

$D_{2}\left(a, C_{1}\right) \equiv \int_{0}^{\sqrt{1-C_{1}^{2}}} \mathrm{~d} e \int_{0}^{2 \pi} \mathrm{d} \omega D_{\mathrm{f}}\left(a, C_{1}, e, \omega\right)$.

Analogically we define integrated quantities

$\mathcal{F}_{1}\left(R_{\min } ; a\right) \equiv \frac{1}{D_{1}(a)} \int_{0}^{1} \mathrm{~d} C_{1} \mathcal{F}_{2}\left(R_{\min } ; a, C_{1}\right) D_{2}\left(a, C_{1}\right)$

and

$\mathcal{F}\left(R_{\min }\right) \equiv \int_{a_{\min }}^{a_{\max }} \mathrm{d} a \mathcal{F}_{1}\left(R_{\min } ; a\right) D_{1}(a)$, where

$D_{1}(a) \equiv \int_{0}^{1} \mathrm{~d} C_{1} D_{2}\left(a, C_{1}\right)$.

The function $\mathcal{F}\left(R_{\min }\right)$ can be interpreted as the volume of the loss cone normalised to the phase space volume occupied by the stellar cluster. (The correspondence between $\mathcal{F}$ and the volume of the loss cone would be exact in the case of uniform distribution function $D_{\mathrm{f}}$.)

The integrals (6)-(10) can be carried out analytically in the Keplerian case. In the non-Keplerian case, however, the function $e_{\max }\left(a, C_{1}, e, \omega\right)$ is given in an implicit form that complicates the evaluation of the integrals. Our approach is thus based on direct numerical integration of the equations of motion of suitably chosen trajectories, as described below.

\subsection{Integration of the orbits}

According to the averaging technique (Arnold 1989; Brower \& Clemence 1961), the mean motion is taken over orbital period $P$. This allows us to study the long-term evolution of the osculating parameters, $(e, \omega, a, i)$ in an integrable (Keplerian) potential with a small perturbation. The approach relies on the existence of a third integral of motion (in addition to the energy and $z$-component of the angular momentum), which represents a mean of the perturbing part of the Hamiltonian. The orbital trajectories form a congruence of closed curves in the space of mean eccentricity vs. argument of pericentre.

We can use the averaging technique to find parameters of the orbits that reach high eccentricities. Then, we integrate the equations of motion directly for those highly eccentric orbits. We take accelerations in the form

$\boldsymbol{a}=-\frac{G M_{\bullet}}{r^{3}} \boldsymbol{r}-\frac{G M_{\bullet}}{c^{2} r^{3}}\left[v^{2} \boldsymbol{r}-4(\boldsymbol{r} \cdot \boldsymbol{v}) \boldsymbol{v}-\frac{4 G M_{\bullet}}{r} \boldsymbol{r}\right]-\boldsymbol{\nabla}\left(V_{\mathrm{d}}+V_{\mathrm{c}}\right)$,

assuming that the leading Newtonian term dominates. The bracketed term is the PN1 correction to the gravity of the central mass (see Sect. VII of Damour et al. 1991), while $V_{\mathrm{d}}$ and $V_{\mathrm{c}}$ represent axially and spherically symmetric perturbations, respectively.

Figure 2 shows a set of tracks of different orbits with their corresponding values of $a$ and $C_{1}$ kept fixed. To find these orbits, we assume equations of motion in the form (11). For the gravitational field we take different terms into account, so that the effect of perturbations becomes apparent. Pericentre argument $\omega$ stands as the polar angle, whereas $\rho(e) \equiv(1-\eta)^{1 / 2}$ is the radial coordinate; maximum eccentricity is then on the perimeter of these graphs, $e_{\max }=\left(1-C_{1}^{2}\right)^{1 / 2}=0.995$. Two classes of orbits can be distinguished - those that librate around $\omega=\pi / 2$ (or $\omega=3 \pi / 2$ ) and circulating orbits for which the whole range of $\langle 0,2 \pi\rangle$ is allowed. The most eccentric orbits attain their maximum eccentricity $e_{\max }$ at $\cos \omega=0$ in the outer circulating region, as is evident from the plots. Hence, to evaluate the fraction $\mathcal{F}_{2}\left(R_{\min } ; a, C_{1}\right)$, we integrate the orbit evolution starting from $(e, \omega) \equiv\left(e_{\min }, \pi / 2\right)$. The result of integration determines a boundary of the region occupied by trajectories with the maximum eccentricity $\geq e_{\min }$. A topology of the contour plot is crucial for the orbit behaviour.

The shaded area shows an intersection of the loss-cone with the $\left(a, C_{1}\right)$ plane. In the example of Fig. 2 we set $e_{\min }=0.989$ and highlight the corresponding contour. This defines the boundary of the shaded area within which every orbit reaches $e>e_{\min }$ 

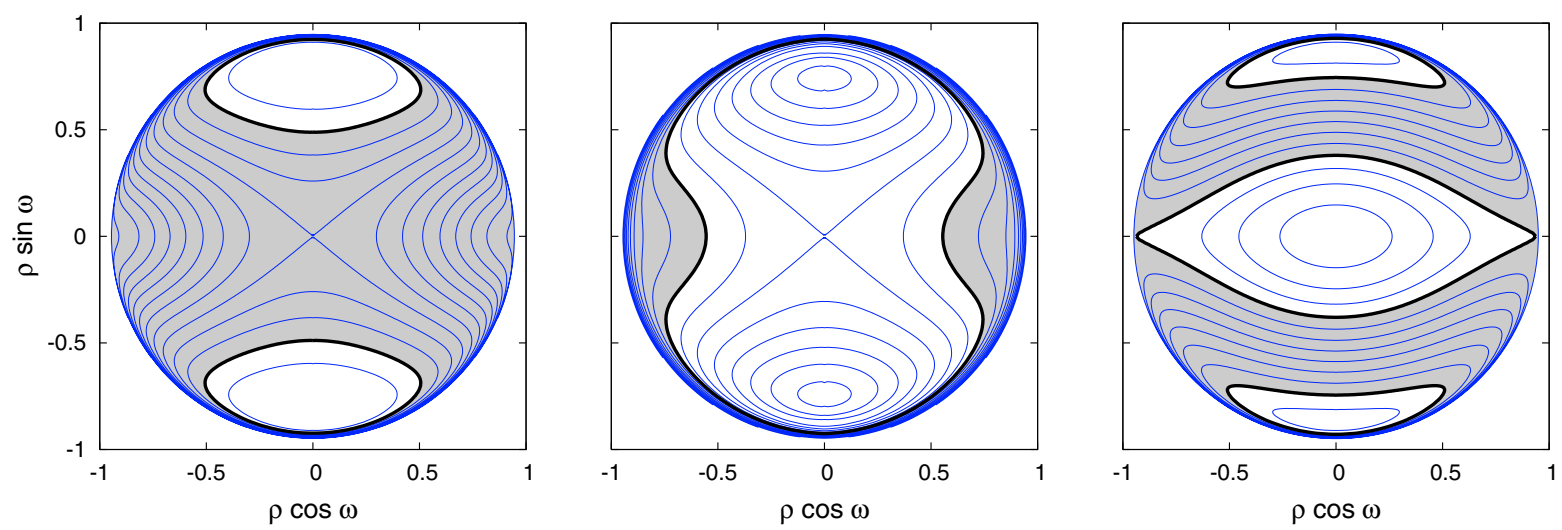

Fig. 2. Structure of the orbits is shown in the $(\rho, \omega)$ polar plot according to Eq. (11). In the left panel, only the dominating Keplerian field and the axisymmetric $\boldsymbol{\nabla} V_{\mathrm{d}}$ perturbation are considered. In the middle panel, the PN1 term of the central field is added. In the right panel, both PN1 of the centre and $\nabla V_{\mathrm{c}}$ of the cluster are considered. High-eccentricity orbits pass through the shaded regions. Common values of the parameters are $M_{\mathrm{c}}\left(R_{\mathrm{h}}\right)=M_{\mathrm{d}}=0.01 M_{\bullet}, R_{\mathrm{d}}=R_{\mathrm{h}}=10^{5} R_{\mathrm{g}}, C_{1}=0.1$, and $a=0.4 R_{\mathrm{d}}$.

at some moment of its evolution. In other words, this area contains highly eccentric orbits and it defines the size and the shape of the loss cone. Having the loss-cone boundary defined, we integrate $D_{\mathrm{f}}\left(a, C_{1}, e, \omega\right)$ over the shaded region and obtain $\mathcal{F}_{2}\left(R_{\min } ; a, C_{1}\right)$. Finally, we evaluate this function on a grid in the $\left(a, C_{1}\right)$ space and integrate it numerically to find $\mathcal{F}_{1}\left(R_{\min } ; a\right)$ and $\mathcal{F}\left(R_{\text {min }}\right)$.

The three panels exhibit different possible topologies of the tracks of orbits that arise due to the perturbing forces. In order to construct these plots, the central Keplerian potential was superposed with the perturbation $\nabla V_{\mathrm{d}}$ (left); with PN1 correction and $\boldsymbol{\nabla} V_{\mathrm{d}}$ terms added (middle); and with PN1 correction and $\boldsymbol{\nabla}\left(V_{\mathrm{d}}+V_{\mathrm{c}}\right)$ terms taken into account (right).

In a conservative system, which is what we consider in Fig. 2, star tracks remain attached to the contours. However, additional dissipative processes might allow adiabatic evolution of the orbital parameters, so that stars can slide gradually across the level surfaces on very long time scales. This may cause a decay of the orbits and help to bring stars to the centre. We do not consider such effect here, but see Šubr \& Karas (2005) where Kozai's resonance mechanism and the hydrodynamical (dissipative) drag are both taken into account.

\section{Results}

As mentioned above, Kozai's resonance mechanism is active when the Newtonian central potential is perturbed by an axisymmetric term, i.e. the disc potential in our case. However, the effect is known to be fragile with respect to various other perturbations that may influence the motion of stars; namely, it is suppressed when the central field is non-Newtonian, leading to the precession of the orbits. Therefore we want to clarify whether Kozai's mechanism is still relevant for the long-term dynamics of nuclear stars even if relativistic pericentre advance and the effect of the nuclear cluster are taken into account.

\subsection{Fractional probabilities}

First we neglect all perturbing terms in the gravitational field and assume purely Keplerian motion. The maximum eccentricity along each trajectory is simply $e_{\max }\left(a, C_{1}, e, \omega\right) \equiv e=$ const.
Assuming the distribution (1), fractional probabilities $\mathcal{F}_{i}$ can then be written in the explicit form:

$\mathcal{F}_{2}\left(R_{\min } ; a, C_{1}\right)=\frac{\eta_{\min }-C_{1}}{1-C_{1}} \Theta\left(\eta_{\min }-C_{1}\right)$,

where $\eta_{\min } \equiv\left(1-e_{\min }^{2}\right)^{1 / 2}$,

$\mathcal{F}_{1}\left(R_{\min } ; a\right)=\eta_{\min }^{2}=\frac{2 R_{\min }}{a}-\frac{R_{\min }^{2}}{a^{2}}$

$$
\begin{aligned}
\mathcal{F}\left(R_{\min }\right) & =\frac{10 R_{\min }\left(a_{\max }^{1 / 4}-a_{\min }^{1 / 4}\right)+\frac{5}{3} R_{\min }^{2}\left(a_{\max }^{-3 / 4}-a_{\min }^{-3 / 4}\right)}{a_{\max }^{5 / 4}-a_{\min }^{5 / 4}} \\
& \simeq 10 R_{\min } a_{\max }^{-1} .
\end{aligned}
$$

To derive the approximation (15) we assumed $R_{\min } \ll a_{\min } \ll$ $a_{\max }$.

Under an axially symmetric perturbation of the central potential, the total angular momentum of orbits is no longer conserved; nevertheless, its $z$-component remains an integral of motion. This results in oscillations of eccentricity. An upper estimate of the corresponding probabilities can be derived from the loss-cone condition, $L_{z}<L_{\max }$, which can be expressed as $e_{\max }\left(a, C_{1}, e, \omega\right)=\left(1-C_{1}^{2}\right)^{-1 / 2}$. Then,

$\mathcal{F}_{2}\left(R_{\min } ; a, C_{1}\right)=\Theta\left(\eta_{\min }-C_{1}\right)$,

$\mathcal{F}_{1}\left(R_{\min } ; a\right)=2 \eta_{\min }-\eta_{\min }^{2} \simeq 2\left(2 R_{\min } a^{-1}\right)^{1 / 2}$,

and

$\mathcal{F}\left(R_{\min }\right) \simeq \frac{10}{3} \frac{\left(2 R_{\min }\right)^{1 / 2}\left(a_{\max }^{3 / 4}-a_{\min }^{3 / 4}\right)}{a_{\max }^{5 / 4}-a_{\min }^{5 / 4}} \simeq 4.71\left(\frac{R_{\min }}{a_{\max }}\right)^{1 / 2}$

The last term on the right-hand side gives values two to three orders of magnitude higher than those that follow from Eq. (15). The estimate (18) is good provided the Kozai mechanism is the only one determining the orbital changes. However, this resonance mechanism may be diminished by other perturbations, so the actual value of $\mathcal{F}\left(R_{\text {min }}\right)$ should be somewhere in between the two estimates. We now discuss the importance of damping effects. 

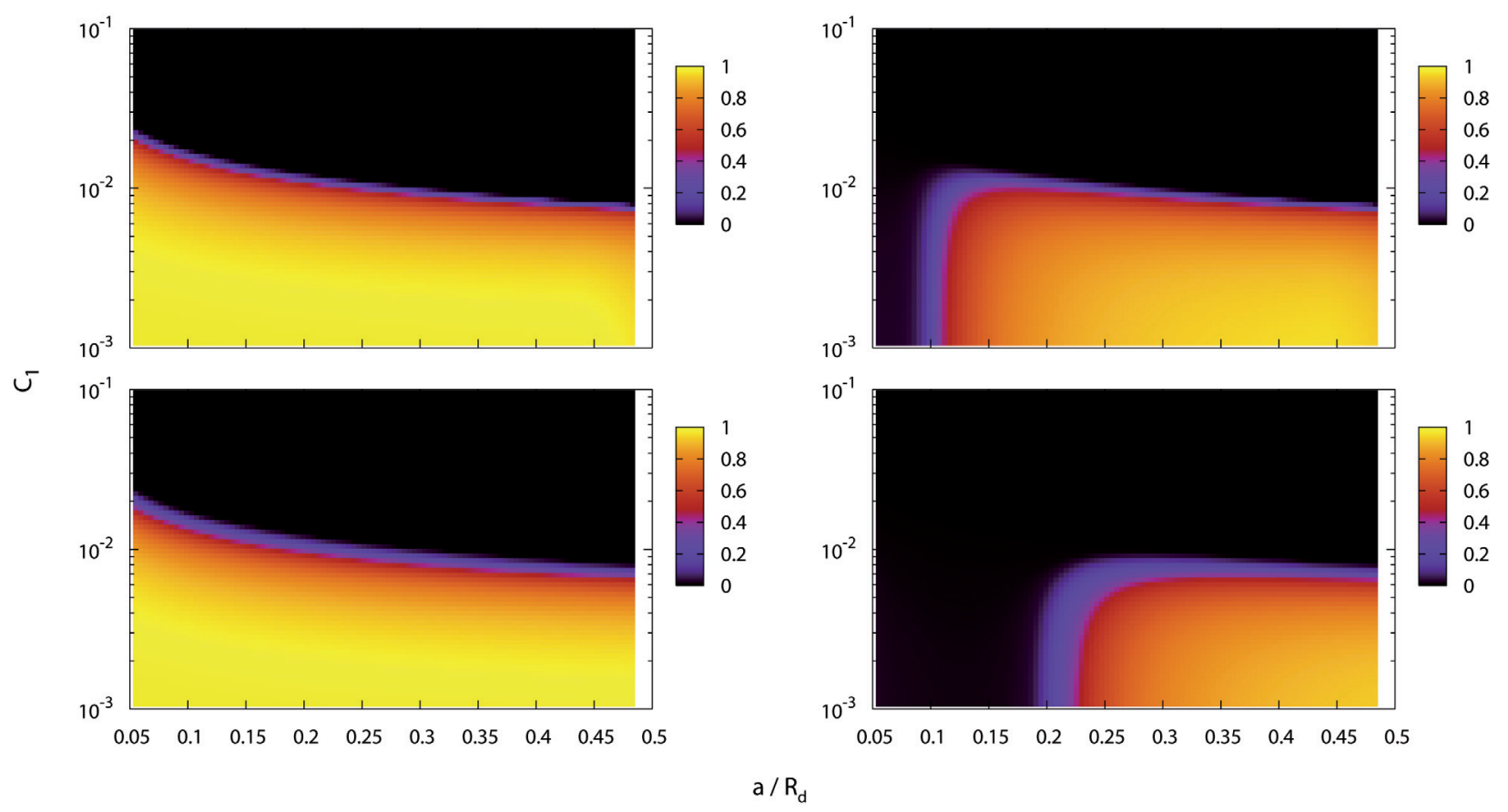

Fig. 3. The clear distinction between the cases with (on the right) and without (on the left) the relativistic pericentre advance taken into account. We draw the fraction $\mathcal{F}_{2}\left(R_{\min } ; a, C_{1}\right)$ of stars from the ensemble (1) that plunge below $R_{\min }=100 R_{\mathrm{g}} ; a$ and $C_{1}$ are constants of motion. The central black hole mass $M_{\bullet}=3.6 \times 10^{6} M_{\odot}$, the disc mass $M_{\mathrm{d}}=0.1 M_{\bullet}$. In the bottom panels we assumed the central gravitational field perturbed by a narrow ring. In the upper panels, the case of a constant-density disc was considered. The left column is for the Newtonian potential of the central source, while we employed the post-Newtonian (PN1) model on the right side. The value of $\mathcal{F}_{2}$ is encoded by different levels, ranging from zero to unity.

The relativistic advance of pericentre has an overall tendency decrease the maximum eccentricity over the Kozai cycle. Below a certain threshold value of $a$, the maximum eccentricity is not sufficient to bring stars inside the tidal radius; the loss-cone condition is then $L<L_{\max }$. We estimate this terminal value as

$a_{\mathrm{t}}^{7} \simeq \frac{32}{9} R_{\mathrm{d}}^{6} R_{\mathrm{g}}^{2} R_{\min }^{-1} \mu^{-2}$

(see Appendix A). For $a>a_{\mathrm{t}}$ the maximum eccentricity allowed by the relativistic corrections is high enough that the pericentre goes below $R_{\min }$. Hence, the fraction of tidally disrupted stars is determined solely by the estimate (13) for $a<a_{\mathrm{t}}$, while the fraction increases abruptly above $a_{\mathrm{t}}$ and saturates almost at the value (17).

\subsection{Stars plunging below a threshold radius}

In Fig. 3 we plot the fractional probability $\mathcal{F}_{2}\left(R_{\min } ; a, C_{1}\right)$, as we obtained it numerically. Comparison between different panels clearly demonstrates the reason for the concern that the pericentre advance of stellar orbits might completely erase the effect of resonance. The figure also shows how sensitive the value of $\mathcal{F}_{2}$ is with respect to the pericentre precession and the eccentricity oscillations - the two effects which act against each other. Notice a sharp drop of $\mathcal{F}_{2}$ below a certain critical value of $a=a_{\mathrm{t}}$ (in the panels on the right side). The break emerges once the pericentre advance is taken into account; it does not occur in the left panels because these were computed in purely Newtonian gravity.

One can see that Kozai's mechanism indeed sets stars on eccentric trajectories reaching small radii and that the efficiency of the process increases with $a$ decreasing down to a certain limiting value. We considered both the Newtonian and the postNewtonian models of the central field, so the effect of the relativistic pericentre advance can be distinguished. We find that $\mathcal{F}_{2}$ is raised by a factor of $\simeq 100$ due to the Kozai mechanism and it drops at a small semi-axis; the exact value of $a$ where that break occurs depends on the adopted form of the perturbing potential. Comparison of the panels reveals that the disc-like source of the gravity competes more successfully with the relativistic effect than a narrow ring of the same mass.

The mean potential of the extended cluster of stars also causes secular precession of the orbits, but this time it alters the results in a different way than the relativistic pericentre advance by attenuating the Kozai oscillations over the whole range of semi-major axes. Nevertheless, it still allows a substantial fraction of orbits to reach high eccentricities.

The fraction $\mathcal{F}_{1}\left(R_{\mathrm{t}} ; a\right)$ is shown in Fig. 4. We find the damping effects to be weakened if the mass of the axisymmetric component is higher, as expected. By increasing the mass parameter $\mu$ the curve of $\mathcal{F}_{1}\left(R_{\mathrm{t}} ; a\right)$ gradually gets shifted to higher values. Simultaneously, $a_{\mathrm{t}}$ is diminished. As a consequence of these dependencies, more stars reach the tidal radius for lower masses of the central body than for higher masses. We assume solar-type stars $\left(M_{\star}=1 M_{\odot}\right.$ and $\left.R_{\star}=1 R_{\odot}\right)$ in the evaluation of $R_{\mathrm{t}}$ in Eq. (5).

Finally, the overall fraction $\mathcal{F}\left(R_{\min }\right)$ is shown in Fig. 5. The limits on the range of semi-major axes are set mainly by numerical arguments: the lower limit results from a sharp increase in the Kozai time $T_{\mathrm{K}}$ when $a$ is decreased. Below $a_{\text {min }}=0.04 R_{\mathrm{h}}$, the orbits contribute only marginally to the tidal disruption rate. The upper limit has to be set due to limitations in our approach, because at $r \simeq R_{\mathrm{d}}$ the orbits exhibit chaotic motion and no longer follow the curves of Fig. 2. We assume that these orbits will lead to the disruptions and, therefore, results presented here should be considered as lower estimates. 

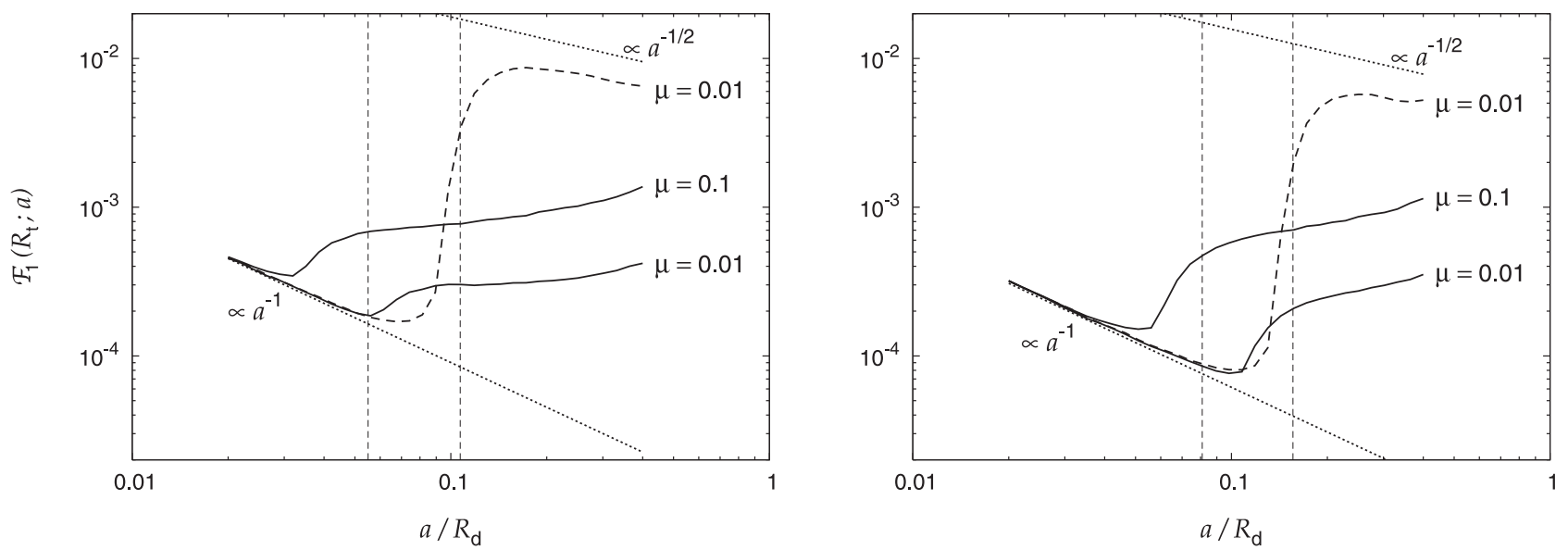

Fig. 4. Fraction $\mathcal{F}_{1}\left(R_{\mathrm{t}} ; a\right)$ of stars plunging below the black hole tidal radius. Stellar orbits are perturbed by a massive ring of radius $R_{\mathrm{d}}$ and mass $M_{\mathrm{d}}=\mu M_{\bullet}$ (indicated with curves). The dashed line is for the PN1 approximation of the central mass gravity field; the effect of the outer stellar cluster is neglected. Solid lines represent two cases in which the cluster is taken into account (they differ by the disc mass parameter $\mu$ ). Dotted are two power-law lines corresponding to the lower and the upper analytical estimates, Eqs. (13) and (17), respectively. Vertical dashed lines indicate the values of $a_{\mathrm{t}}$ (given by Eq. (19) with $\mu=0.01$ and 0.1 , respectively); these are critical semiaxis values below which Kozai's phenomenon is suppressed. The eccentricity oscillations persist for $a>a_{\mathrm{t}}$. Parameter values assumed in this plot are: in the left panel $M_{\bullet}=10^{4} M_{\odot}$; in the right panel $M_{\bullet}=10^{5} M_{\odot}$.

Line types help us to distinguish the importance of different effects in this plot. In particular:

(i) Kozai's oscillations raise the value of $\mathcal{F}$ with respect to an unperturbed $(\mu=0)$ case;

(ii) PN1 corrections to the central (Keplerian) potential reduce $\mathcal{F}$ by a factor $\simeq 2 \div 5$ (the expected rate of tidal disruptions is diminished accordingly);

(iii) precession due to self-gravity of the cluster decreases $\mathcal{F}$ further down by another factor $\simeq 10$.

As in the previous figures, it is clearly visible that the potential of the extended source, i.e. the disc, competes more successfully against damping than does the ring. Increasing the mass of the axial perturbation not only raises $\mathcal{F}$ to higher values, but it also changes the slope. That tendency can be attributed to a higher terminal value $a_{\mathrm{t}}$ of the semi-major axis, above which the relativistic damping is negligible.

The effect of the relativistic pericentre advance increases with the mass of the black hole. The right panels of Fig. 5 demonstrate that $\mathcal{F}$ is anti-correlated with $M_{\bullet}$. and grows towards less massive black holes (i.e. when going from $\mathrm{SMBH}$ to IMBH).

\section{Discussion}

We examined the idea of Kozai's effect assisted by an accretion disc as the mechanism enhancing the disruption rate of stars by a central black hole. This process is more efficient for intermediate-mass black holes than for supermassive ones. In our calculations we used the PN1 approximation of the central field. We also performed these calculations within the pseudoNewtonian (Paczyński-Wiita 1980) framework (as a check of the orbits very near horizon) with very similar conclusions.

The results for $\mathcal{F}\left(R_{\mathrm{t}}\right)$ can be directly interpreted as the fraction of stars populating the loss-cone when an axisymmetric perturbation is applied on the cluster. We suggest that this picture is relevant in a system where gas clouds form an embedded disclike structure with a non-negligible total mass near the sphere of dominance of a central black hole.

\subsection{Disturbing processes}

The view presented here is valid as long as other processes, acting on time scales shorter than $T_{\mathrm{K}}$, do not manage to expel the stars from the effective loss cone. This would inhibit the Kozai process. One of the generic processes that may interfere with the influence of the axisymmetric perturbation is the two-body relaxation within the stellar cluster.

Gravitational relaxation brings the diffusion time scale into the problem, $T_{\theta} \equiv \theta_{\mathrm{lc}}^{2} T_{\mathrm{r}}$, where

$\theta_{\mathrm{lc}}=\sqrt{R_{\mathrm{t}} / r} \simeq \sqrt{\mathcal{F}\left(R_{\mathrm{t}}\right)} \ll 1$

is an angular extent of the loss cone and

$T_{\mathrm{r}}=\frac{\sigma^{3}}{G^{2} C \ln \Lambda M_{*}^{2} n_{*}}$

Here, $n_{*}$ is the number density of the stellar system, $\ln \Lambda$ is the Coulomb logarithm, and $C$ is a constant (in usual notation, $C \ln \Lambda \sim 10^{2}$; Spitzer 1987). In a cusp described by the Bahcall-Wolf distribution, velocity dispersion is comparable to Keplerian velocity, and the number density of stars decreases with radius as $n_{*}(r) \propto r^{-7 / 4}$. Characterising the number density by the stellar mass $M_{\mathrm{c}}$ enclosed within the radius $R_{\mathrm{h}}$, we get

$$
T_{\mathrm{r}} \simeq 10^{6} M_{4}^{2}\left(\frac{M_{\mathrm{c}}}{M_{\bullet}}\right)^{-1}\left(\frac{M_{*}}{M_{\odot}}\right)^{-2}\left(\frac{R_{\mathrm{h}}}{10^{8} R_{\mathrm{g}}}\right)^{5 / 4}\left(\frac{r}{10^{8} R_{\mathrm{g}}}\right)^{1 / 4} \mathrm{yr} .
$$

The effective loss cone invoked in Sect. 2.1 generally exceeds $\theta_{\mathrm{lc}}$. From Fig. 5 we see that its volume $\mathcal{F}\left(R_{\mathrm{t}}\right)$ is (for $\mu=0.1$ and a ring source of the perturbation) a factor $>10$ larger than it is in an unperturbed system. Hence, appropriate $\theta_{\mathrm{lc}}^{\prime}$ must be by a factor $\gtrsim 3$ larger than $\theta_{\mathrm{lc}}$. However, the geometry of the effective loss cone is more complicated. In particular, it is narrower at $\omega=\pi / 2$ where it actually coincides with the boundary of the classical loss cone. Therefore, at $\omega$ corresponding to the minimum eccentricity, the new loss cone must be sufficiently wider so that its volume can exceed the classical one by a factor of ten. We estimate $\theta_{\mathrm{lc}}^{\prime} \simeq 10 \theta_{\mathrm{lc}}$, which is also in accordance with our experience that the pericentre value on the boundary orbit of the effective loss cone typically changes by more than two orders 

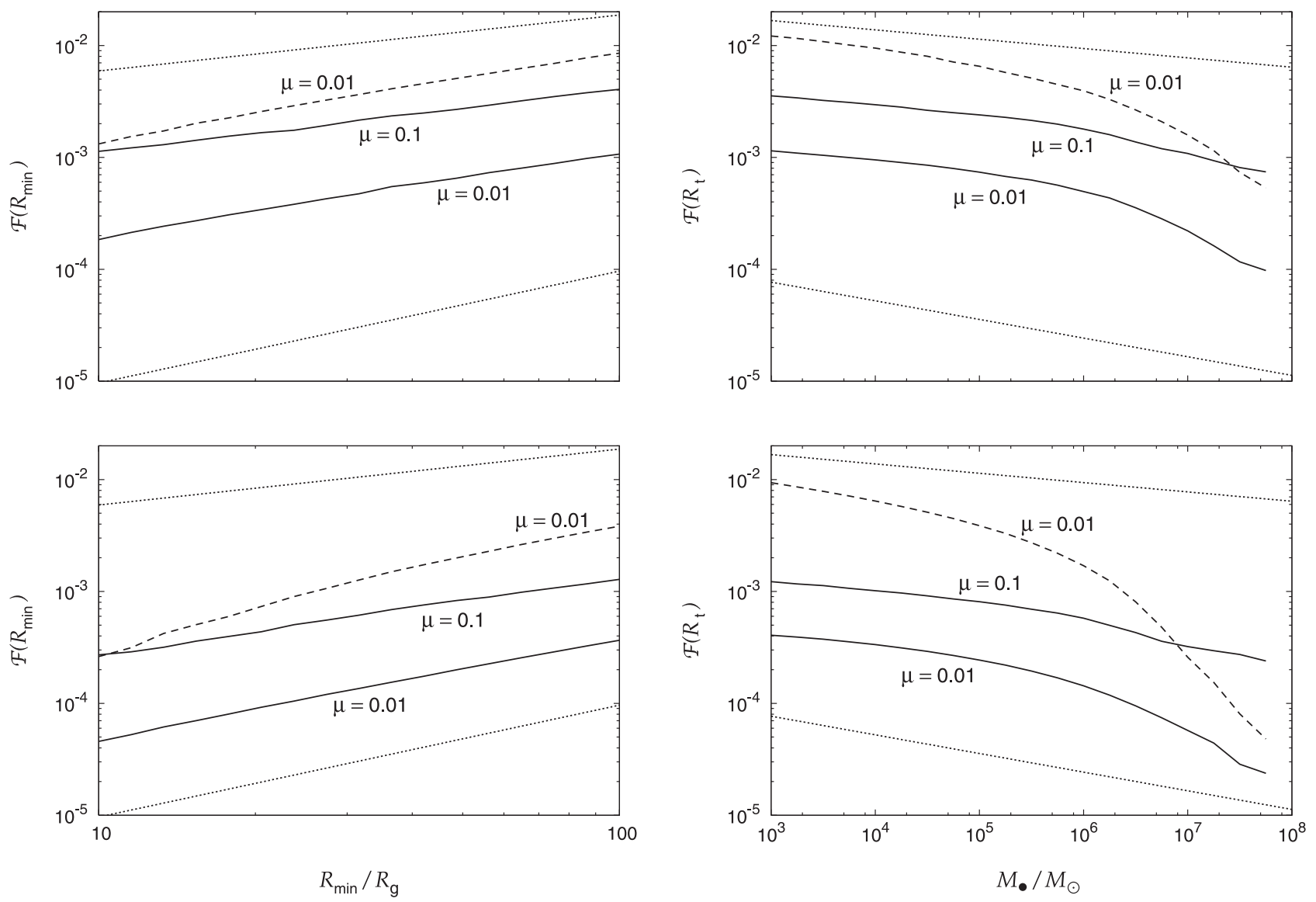

Fig. 5. Fraction $\mathcal{F}\left(R_{\min }\right)$ of stars reaching the pericentre below a given radius $r=R_{\min }$. We assumed perturbing potentials $V_{\mathrm{d}}(R, z)$ of the disc with constant surface density (top panels) and a narrow ring (bottom panels). Left: the functional dependence of probability $\mathcal{F}\left(R_{\text {min }}\right)$ (solid lines). Parameters compatible with Sgr $A^{\star}$ were assumed: $M_{\bullet}=3.5 \times 10^{6} M_{\odot}, 0.01 \leq \mu \leq 0.1, R_{\mathrm{h}} \simeq R_{\mathrm{d}}=1.2 \times 10^{7} R_{\mathrm{g}}=2 \mathrm{pc}$, and $0.04 R_{\mathrm{h}} \leq a \leq 0.4 R_{\mathrm{h}}$. Right: the mass dependence, $\mathcal{F}\left(R_{\mathrm{t}}\left(M_{\bullet}\right)\right)$, computed according to Eqs. (2) and (5). In all panels dotted lines correspond to the analytical estimates (14) and (18); values given by the latter correspond within a factor of $\lesssim 2$ to the case omitting both the relativistic pericentre advance and the stellar cluster reducing the Kozai oscillations. Dashed lines represent models with only the relativistic precession switched on $(\mu=0.01)$, while the solid lines also include the precession from the cluster potential (two cases are shown, $\mu=0.01$ and 0.1 ).

of magnitude. The time scale on which stars diffuse across the effective loss-cone is then

$T_{\theta}^{\prime} \simeq 100 T_{\theta} \simeq 0.04 M_{4}^{3 / 2}\left(\frac{M_{\mathrm{c}}}{M_{\bullet}}\right)^{-1}\left(\frac{M_{*}}{M_{\odot}}\right)^{-1}\left(\frac{r}{R_{\mathrm{h}}}\right)^{-9 / 4} P$.

By comparing $T_{\mathrm{K}}$ and $T_{\theta}^{\prime}$, we deduce that for $M_{4} \gtrsim 10$ the Kozai mechanism affects the angular momentum on a significantly shorter time scale, so we may consider the effective loss cone to be emptied by this process.

For lower BH masses, the interplay of the Kozai mechanism and the two-body relaxation is more complicated; however, we still expect that the systematic character of the angular momentum changes because the Kozai mechanism will enhance the rate of tidal disruptions. A definitive study of these systems is probably not possible without employing high-precision $N$-body integrators.

We imagine that consumption of stars by the central IMBHs is a transient process that enhances their activity during those periods when stars are supplied into the loss cone and then efficiently brought onto eccentric orbits. Permanent replenishment of this larger loss cone is not critical for the process considered here. Nevertheless, we may assume that the larger loss cone is replenished more frequently (also by other processes than the steady state relaxation - for example the effect of the Brownian motion of the central black hole, or a secondary black hole can contribute). However, details of the loss-cone refilling are beyond the scope of this paper. It will be interesting to compare these speculations with the results of $\mathrm{N}$-body simulations that have been recently applied to exploring the tidal processes and their effect on IMBHs formation and feeding in dense star clusters (see Baumgardt et al. 2006).

To achieve a more complete description, one has to invoke other diffusive processes acting with the gravitational relaxation (e.g. the hydrodynamical drag by the disc gas that continuously helps refilling the loss-cone; see e.g. Karas \& Šubr 2001 for relevant time scales) but, again, this is beyond the scope of our paper.

\subsection{Dependence on the black hole mass}

Let us consider two types of object that differ from each other by the black hole mass: (i) a supermassive black hole in the Galaxy center and (ii) hypothetical intermediate-mass black holes that might reside in cores of dense star clusters.

Precise tracking of the proper motion of individual stars is currently possible within an arcsecond area around the Galaxy centre (Genzel et al. 2003; Ghez et al. 2003), so the idea of applying our calculation to $\mathrm{Sgr} \mathrm{A}^{\star}$ is quite natural and raises a question about the origin of various perturbations that may act on stellar motion. Currently, $M_{\mathrm{d}} \simeq 0.1 M_{\bullet}$ can be set as an upper 
estimate of the mass of the molecular circumnuclear disc extending from $\sim 1.5$ pc to $3 \div 4$ pc (Christopher et al. 2005), i.e. on the outer edge of the black hole's sphere of influence. This is compatible with a narrow ring of radius $R_{\mathrm{d}}=R_{\mathrm{h}} \simeq 2 \mathrm{pc}$, so we can directly evaluate the expected number of tidal disruptions. From Fig. 5 we read that the fraction $\simeq 5 \times 10^{-4}$ of the total number of stars in the cluster with $a_{\max }<0.4 R_{\mathrm{d}}$ exhibit sufficiently large eccentricity oscillations. This corresponds to a mass in stars of about $\simeq 0.1 M_{\bullet} \simeq 3 \times 10^{5} M_{\odot}$, from which we obtain $\simeq 150$ tidal disruption events per $T_{\mathrm{K}} \simeq 10 \mathrm{Myr}$.

So far, we have assumed that the source of the gravitational perturbation was a gaseous disc or a torus; however, its origin could be different. In the $\operatorname{Sgr} \mathrm{A}^{\star}$ there is a disc of young stars (Levin \& Beloborodov 2003; Paumard et al. 2006) and, possibly, a remnant gas from which these stars were born. To model their effect we consider a thin disc of mass $0.01 M_{\bullet}$ and surface density $\propto R^{-2}$ extending between $0.03 \div 0.3 \mathrm{pc}$. We find that $\simeq 2 \%$ of stars from the region $<0.3 \mathrm{pc}$ can undergo the oscillations that bring them down to $R_{\mathrm{t}}$. This means that, during the period of $T_{\mathrm{K}} \lesssim 1 \mathrm{Myr}$ after the formation of the gaseous/stellar disc, up to $\simeq 100$ more tidally disrupted stars may have occurred compared to the result of calculations neglecting the effect. The phase of enhanced disruption rate may become prolonged if the orientation of the disc varies in time. This could be caused by precession in the outer galactic potential.

In the case of IMBHs, the resonance mechanism complements the role of stellar encounters in the process of feeding the black hole. If the total mass of the cluster is about $M_{\bullet} \simeq 10^{4} M_{\odot}$, we expect the enhancement of the disruption rate $\simeq 1 M_{\odot}$ per $10^{3} \mathrm{yr}$ (with $\mu=0.1$ ). Disrupted stars then provide material for accretion, thereby triggering a transient luminous phase of the object.

We note that the model has only formal validity near the lower end of the black-hole mass range because in this case the accretion rate of $\simeq 10^{-3} M_{\bullet} \mathrm{yr}^{-1}$ is comparable to the mass carried by individual stars; other processes must be important under such circumstances (which is consistent with the expectation that the accretion of stellar material onto IMBH is a non-steady process). Therefore, the scenario outlined above conforms to the assumption that ULXs are a transient phenomenon for which accretion of the surrounding medium is essential.

\section{Conclusions}

Disruption of stellar bodies and subsequent accretion of the remnant gas are among likely mechanisms feeding black holes that are embedded in a dense cluster. We discussed one of the channels that may contribute to this process. Kozai's mechanism can enhance the rate of such events, trigger the episodic gas supply onto the black hole, and, consequently, strengthen the activity of the system by raising the accretion rate. The process acts on the characteristic time scale of the Kozai cycle - typically $T_{\mathrm{K}} \simeq 10^{5} \mathrm{yr}$ for IMBHs - during which the loss cone is depleted.

Stars on highly elongated orbits are susceptible to tidal disruption and hence provide a natural source of material to replenish the inner disc. We have demonstrated that this phenomenon operates in the system even if it is disturbed by the relativistic pericentre precession and by the gravity of the nuclear cluster. Therefore we can conclude that the presence of a gaseous disc of a small but non-zero mass, $0<M_{\mathrm{d}}<M_{\bullet}$, helps to drag stars to the black hole, thereby feeding the centre and simultaneously providing material that sustains and replenishes the disc itself.
Acknowledgements. We thank Marc Freitag for helpful discussions about the gravitational influence of the cluster and Holger Baumgardt for the possible role of IMBHs in ULXs. This work was supported by the Centre for Theoretical Astrophysics in Prague (ref. LC06014) and the DFG Priority Program 1177 "Witnesses of Cosmic History: Formation and Evolution of Black Holes, Galaxies and Their Environment". The Astronomical Institute of the Academy of Sciences is financed via Ministry of Education project ref. AV0Z10030501. VK gratefully acknowledges the continued support from the Czech Science Foundation (ref. 205/07/0052).

\section{Appendix A: The role of GR pericentre advance}

Here we want to estimate the region of the parameter space where the general relativistic pericentre advance dominates the Kozai effect. The quadrupole approximation leads to evolutionary equations (Kiseleva et al. 1998; Blaes et al. 2002):

$$
\begin{aligned}
T_{\mathrm{K}} \eta \frac{\mathrm{d} i}{\mathrm{~d} t} & =-\frac{15}{8} e^{2} \sin 2 \omega \sin i \cos i, \\
T_{\mathrm{K}} \eta \frac{\mathrm{d} e}{\mathrm{~d} t} & =\frac{15}{8} e \eta^{2} \sin 2 \omega \sin ^{2} i, \\
T_{\mathrm{K}} \eta \frac{\mathrm{d} \omega}{\mathrm{d} t} & =\frac{3}{4}\left\{2 \eta^{2}+5 \sin ^{2} \omega\left[e^{2}-\sin ^{2} i\right]\right\}+T_{\mathrm{K}} \frac{R_{\mathrm{g}}}{a \eta} \frac{6 \pi}{P} .
\end{aligned}
$$

The last term on the right-hand side of Eq. (A.3) includes the relativistic pericentre advance.

Equations (A.1)-(A.3) imply conservation of

$C_{1}=\eta \cos i$

and

$C_{2}=\left(5 \sin ^{2} i \sin ^{2} \omega-2\right) e^{2}-\frac{8 R_{\mathrm{d}}^{3} R_{\mathrm{g}}}{\mu \eta a^{4}}$

Solutions of two types can be found - circulating vs. librating depending on the motion constants $a, C_{1}$, and $C_{2}$. The circulation region always exists, while the libration region typically occurs for low values of $C_{1}$. The two categories are separated by the separatrix curve that passes through the $e=0$ point in the plane of $(e, \omega)$ polar coordinates. This can be used to determine the value of $C_{2}$ on the separatrix:

$C_{2, \mathrm{~s}}=-\frac{8 R_{\mathrm{d}}^{3} R_{\mathrm{g}}}{\mu a^{4}}$

Maximum eccentricity on the separatrix is given by the equation

$5\left(1-\frac{C_{1}^{2}}{\eta_{\mathrm{s}}^{2}}\right)-\frac{8 R_{\mathrm{d}}^{3} R_{\mathrm{g}}}{\mu a^{4}} \frac{1}{\eta_{\mathrm{s}}\left(1+\eta_{\mathrm{s}}\right)}=2$,

which for $\eta_{\mathrm{s}} \ll 1$ simplifies to the form

$3 \eta_{\mathrm{s}}^{2}-\frac{8 R_{\mathrm{d}}^{3} R_{\mathrm{g}}}{\mu a^{4}} \eta_{\mathrm{s}}=5 C_{1}^{2}$

We are interested in small $C_{1}$. For $C_{1} \lesssim 0.01$, the circulating solutions cover about half of the $(e, \omega)$ parameter space. These orbits acquire eccentricities higher than $e_{\mathrm{s}}$ at some moment during the orbit evolution. Hence, when $e_{\mathrm{s}}\left(a, C_{1}\right)>1-a / R_{\min }$, the fraction $\mathcal{F}_{2}\left(R_{\min } ; a, C_{1}\right)$ is close to the corresponding estimate (16), which assumes that all orbits with given values of $a$ and $C_{1}$ reach the pericentre below $R_{\min }$. Setting $\eta_{\mathrm{s}}^{2}=1-e_{\min }^{2} \simeq 2 R_{\min } / a$, Eq. (A.8) gives an implicit formula for the boundary of these two regions in the $\left(a, C_{1}\right)$ plane. We can solve (A.8) for $C_{1}=0$ to determine the terminal value (19) of semi-major axis $a_{\mathrm{t}}$. Below this value, the relativistic pericentre advance inhibits the Kozai mechanism. 


\section{References}

Alexander, T. 2005, Phys. Rep., 419, 65

Arnold, V. I. 1989, Mathematical Methods of Classical Mechanics (Berlin: Springer-Verlag)

Bahcall, J. N., \& Wolf, R. A. 1976, ApJ, 209, 214

Baumgardt, H., Hopman, C., Portegies Zwart, S., \& Makino, J. 2006, MNRAS, 372,467

Begelman, M., \& Rees, M. J. 1978, MNRAS, 185, 847

Blaes, O., Lee, M. H., \& Socrates, A. 2002, ApJ, 578, 775

Brower, D., \& Clemence, G. 1961, Methods of Celestial Mechanics (New York: Academic Press)

Christopher, M. H., Scoville, N. Z., Stolovy, S. R., \& Yun, M. S. 2005, ApJ, 622, 346

Colbert, E. J. M., \& Mushotzky, R. F. 1999, ApJ, 519, 8

Damour, T., \& Soffel, M., \& Xu, C. 1991, Phys. Rev. D, 43, 3273

Fabian, A. C., Ross, R. R., \& Miller, J. M. 2004, MNRAS, 355, 359

Fabbiano, G. 2006, ARA\&A, 44, 323

Frank, J., \& Rees, M. J. 1976, MNRAS, 176, 633

Gebhardt, K., Rich, R. M., \& Ho, L. C. 2005, ApJ, 634, 1093

Genzel, R., Schödel, R., Ott, T., Eisenhauer, F., et al. 2003, ApJ, 594, 812

Gerssen, J., van der Marel, R. P., Gebhardt, K., et al. 2002, AJ, 124, 3270

Ghez, A. M., Becklin, E. E., Duchêne, G., et al. 2003, Astron. Nachr., 324, 527

Gonçalves, A. C., \& Soria, M. 2006, MNRAS, 371, 673

Gültekin, K., Miller, M. C., \& Hamilton, D. P. 2004, ApJ, 616, 221

Hopman, C., Portegies Zwart, S. F., \& Alexander, T. 2004, ApJ, 604, L101

Huré, J.-M., \& Pierens, A. 2005, ApJ, 624, 289

Ivanov, P. B., Polnarev, A. G., \& Saha, P. 2005, MNRAS, 358, 1361

Karas, V., \& Šubr, L. 2001, A\&A, 386, 686

King, A. R., Davies, M. B., Ward, M. J., Fabbiano, G., \& Elvis, M. 2001, ApJ, 559, L101

Kiseleva, L. G., Eggleton, P. P., \& Mikkola, S. 1998, MNRAS, 300, 292

Kozai, Y. 1962, AJ, 67, 591
Krolik, J. H. 2004, ApJ, 615, 383

Lass, H., \& Blitzer, L. 1983, Celest. Mech. Dyn. Astron., 30, 225

Levin, Y., \& Beloborodov, A. M. 2003, ApJ, 590, L33

Lidov, M. L. 1962, Planet. Space Sci., 9, 719

Madau, P., \& Rees, M. J. 2001, ApJ, 551, L27

Magorrian, J., \& Tremaine, S., 1999, MNRAS, 309, 447

Makishima, K., Kubota, A., Tsunefumi, M., et al. 2000, ApJ, 553, 632

Matsumoto, H., Tsuru, T. G., Koyama, K., et al. 2001, ApJ, 574, L25

McLaughlin, D. E., Anderson, J., Meylan, G., et al. 2006, ApJSS, 166, 249

Merritt, D., \& Wang, J. 2005, ApJ, 621, L101

Miller, M. C., \& Hamilton, D. P. 2002, MNRAS, 330, 232

Miller, J. M., Fabian, A. C., \& Miller, M. C. 2004a, ApJ, 607, 931

Miller, J. M., Fabian, A. C., \& Miller, M. C. 2004b, ApJ, 614, L117

Miniutti, G., Ponti, G., Dadina, M., et al. 2006, MNRAS, 373, L1

Nayakshin, S., \& Sunyaev, R. 2003, MNRAS, 343, L15

Nayakshin, S., Dehnen, W., Cuadra, J., \& Genzel, R. 2005, MNRAS, 366, 1410

Paczyński, B., \& Wiita, P. J. 1980, A\&A, 88, 23

Paumard, T., Genzel, R., Martins, F., et al. 2006, ApJ, 643, 1011

Portegies Zwart, S. F., Baumgardt, H., Hut, P., Makino, J., \& McMillan, S. L. W. 2004, Nature, 428, 724

Spitzer, L. 1987, Dynamical Evolution of Globular Clusters (Princeton: Princeton University Press)

Šubr, L., \& Karas, V. 2005, A\&A, 433, 405

Šubr, L., Karas, V., \& Huré, J.-M. 2004, MNRAS, 354, 1177

Syer, D., Clarke, C. J., \& Rees, M. J. 1991, MNRAS, 250, 505

Tremaine, S., Gebhardt, K., Bender, R., et al. 2002, ApJ, 574, 740

van der Marel, R. P. 2004, in Coevolution of Black Holes and Galaxies, ed. L. C. Ho (Cambridge: Cambridge University Press), 37

Vokrouhlický, D., \& Karas, V. 1993, MNRAS, 265, 365

Vokrouhlický, D., \& Karas, V. 1998, MNRAS, 298, 53

Volonteri, M., \& Rees, M. J. 2005, ApJ, 633, 624

Volonteri, M., Haardt, F., \& Madau, P. 2003, ApJ, 582, 559

Zhao, H., Haehnelt, M. G., \& Rees, M. J. 2002, New Astron., 7, 385 